\title{
Identifying Anthropogenic Uranium Compounds Using Soft X-Ray Near-Edge
}

\section{Absorption Spectroscopy}

Jesse D. Ward, Mark Bowden, C. Tom Resch, Gregory C. Eiden, C. D. Pemmaraju, David Prendergast, Andrew M. Duffin

\begin{abstract}
Uranium ores mined for industrial use are typically acid-leached to produce yellowcake and then converted into uranium halides for enrichment and purification. These anthropogenic chemical forms of uranium are distinct from their mineral counterparts. The purpose of this study is to use soft X-ray absorption spectroscopy to characterize several common anthropogenic uranium compounds important to the nuclear fuel cycle. Chemical analyses of these compounds are important for process and environmental monitoring. X-ray absorption techniques have several advantages in this regard, including element-specificity, chemical sensitivity, and high spectral resolution. Oxygen K-edge spectra were collected for uranyl nitrate, uranyl fluoride, and uranyl chloride, and fluorine K-edge spectra were collected for uranyl fluoride and uranium tetrafluoride. Interpretation of the data is aided by comparisons to calculated spectra. The effect of hydration state on the sample, a potential complication in interpreting oxygen K-edge spectra, is discussed. These compounds have unique spectral signatures that can be used to identify unknown samples.
\end{abstract}

Key Words: uranium; nuclear forensics; X-ray absorption spectroscopy 


\section{Introduction}

By mass, uranium oxides such as pitchblende and uraninite $\left(\mathrm{UO}_{2+x}\right)$ make up the bulk of uranium on Earth, along with a plethora of secondary minerals containing the uranyl ion $\left(\mathrm{UO}_{2}^{2+}\right)[1,2]$. In contrast, uranium halides are not found in nature, but are essential to many uranium purification and enrichment methods. Gaseous diffusion and centrifuge enrichment processes require the conversion of $\mathrm{UO}_{2}$ to uranium hexafluoride $\left(\mathrm{UF}_{6}\right)$, the most volatile known uranium compound. Enriched $\mathrm{UF}_{6}$ is converted back to uranium oxides-typically $\mathrm{UO}_{2}$-for use in power-generating nuclear reactors. Uranium tetrafluoride $\left(\mathrm{UF}_{4}\right)$ is an intermediate in these conversion and deconversion processes, although it also sees practical uses_-for example, as the source material for the production of metallic uranium by the Ames process [3], as the primary fuel of molten salt reactors [4], or as long-term storage for depleted uranium [5]. Uranyl fluoride $\left(\mathrm{UO}_{2} \mathrm{~F}_{2}\right)$, produced by the hydrolysis of $\mathrm{UF}_{6}$, is another intermediate in the deconversion of $\mathrm{UF}_{6}$ to $\mathrm{UO}_{2}$. Solid $\mathrm{UO}_{2} \mathrm{~F}_{2}$ will precipitate out whenever $\mathrm{UF}_{6}$ is allowed to come into contact with moisture [6-8]—for example, when $U_{6}$ gas lines are not sufficiently dry or if a storage cylinder has been structurally compromised.

Methods that can non-destructively detect and characterize these compounds would be highly desirable to monitor process chemistry and to chemically identify any environmental contamination. While a variety of spectroscopic probes exist for detecting uranium, including fluorescence spectroscopy $[9,10]$ and Raman spectroscopy [11, 12], X-ray absorption techniques are advantageous in that they are element-specific and highly sensitive to physical and chemical structure. Soft X-rays-X-rays with an energy 
below $1 \mathrm{keV}$-are particularly useful because absorption edges in this regime show minimal lifetime broadening (0.1-0.3 eV) [13-15], and as a result sharper absorption peaks and greater chemical information can be obtained. The non-destructive nature of X-ray techniques means that subsequent analyses can be performed to obtain further information about the sample (e.g., laser ablation ICP-MS to determine isotopic composition). Furthermore, it is important to develop techniques that can yield spatially resolved information. The ability to extend spectral signatures down to the nanoscale, while not in the scope of the current research, is important because environmental (i.e., samples collected after an accidental release) and IAEA verification samples can be inhomogeneous, with the chemistry of interest at the nanoscale. In the case of X-ray absorption spectroscopy, the incident beam can be focused to a spot size of $10-100 \mathrm{~nm}$, allowing one to map the chemical state of a sample on a fine spatial scale (i.e., on the spatial scale that directly probes the interfaces where the chemical conversion process reactions occur). Scanning transmission X-ray microscopy (STXM) beamlines with a soft X-ray source can produce a focused beam diameter on the order of $20 \mathrm{~nm}$ and can penetrate particles hundreds of nanometers thick, thereby sampling a crucial size range not adequately covered by complementary spectromicroscopic techniques such as electron energy loss spectroscopy (which can produce images with atomic spatial resolution, but is limited by sample thickness) or micro-Raman (which is not limited by sample thickness, but produces images with lower spatial resolution). Although laboratory-based NEXAFS techniques are becoming increasingly common and practical [16-19], access to a synchrotron beamline and the need for an X-ray source that is high- 
flux and broadly tunable between $500-800 \mathrm{eV}$ (see below) ultimately drove us to pursue synchrotron sources specifically.

Most soft X-ray beamlines can probe the uranium N4,5-edges (736.2 eV and $778.3 \mathrm{eV}$ ) directly. The presence of these peaks can serve as a specific marker for uranium in a sample. However, these edges do not contain much chemical information aside from slight shifts in peak positions due to oxidation state, because these peaks result from transitions between nonbonding $d$ and $f$ orbitals [20]. On the other hand, the K-edge absorption spectra of light ligand atoms such as oxygen $(543.1 \mathrm{eV})$ or fluorine $(696.7 \mathrm{eV})$ can exhibit high chemical sensitivity. There is precedent in the literature for using ligand K-edge absorption spectroscopy to obtain information on uranium compounds, e.g., to study $\pi$-bonding in uranyl compounds $[14,21]$ and to study the structures of uranium oxides [22]. Previous work from our group used oxygen near-edge X-ray absorption fine structure (NEXAFS) to classify various uranyl minerals according to their counterion type and to identify uranium oxide samples [23]. These results were extended to classify submicron-diameter schoepite particles at a STXM beamline (ALS beamline 5.3.2.2), to the exclusion of nearby oxygen-containing contaminants.

More generally, for compound identification, it would be desirable to collect X-ray absorption data covering the entire spectral range from the oxygen $\mathrm{K}$-edge at $543.1 \mathrm{eV}$ to the uranium N4,5-edges at $736.2 \mathrm{eV}$ and $778.3 \mathrm{eV}$. This data set would also encompass the fluorine K-edge at $696.7 \mathrm{eV}$. The U N4,5-edges would be used to confirm the specific presence of uranium, compared to other actinides such as plutonium. The oxygen K-edge would be used to classify the uranium mineral according 
to type, and the fluorine K-edge can be used to distinguish important anthropogenic uranium compounds from other fluorine-containing compounds.

The goal of this work is to identify soft X-ray spectroscopic signatures which can distinguish anthropogenic uranium compounds from a background that consists of natural uranium oxides, uranyl minerals, and various other metal oxides and fluorides. To that end, we will focus on two solid uranium fluorides commonly produced in the nuclear fuel cycle—namely, uranyl fluoride and uranium tetrafluoride. We will also analyze a sample of uranyl nitrate $\left(\mathrm{UO}_{2}\left(\mathrm{NO}_{3}\right)_{2}\right)$, which, while not a halide, is a common intermediate in many uranium recovery processes. Finally, we will examine a sample of uranyl chloride $\left(\mathrm{UO}_{2} \mathrm{Cl}_{2}\right) \cdot \mathrm{UO}_{2} \mathrm{Cl}_{2}$ is not commonly used in the nuclear fuel cycle, although uranium compounds can be converted to uranyl chloride prior to electrolytic reduction during uranium purification [24] or as an intermediate in the production of $\mathrm{UF}_{4}$ [25]. Its inclusion is primarily for comparison with $\mathrm{UO}_{2} \mathrm{~F}_{2}$, which is a crucial fuel cycle intermediate and environmental contaminant. Because this work will partly focus on the spectroscopic signatures in the oxygen K-edge, the presence of extraneous water in the sample is a potential complication. Therefore, some effort will be spent to determine the extent of hydration under the vacuum conditions present in a typical soft X-ray beamline and what effect the presence of water would have on the oxygen $\mathrm{K}$-edge. The compounds featured in this work are pure, bulk-quantity samples; however, in the future the spectra measured from these compounds may be applied to identify spectra obtained from particles analyzed by a spatially-resolved technique such as EELS or STXM. For example, the chemical composition of a uranium particle released during a 
nuclear accident [26] would provide data on how the released material weathered in the environment.

\section{Experimental}

All uranium samples—uranyl nitrate $\left(\mathrm{UO}_{2}\left(\mathrm{NO}_{3}\right)_{2}\right)$, uranyl chloride $\left(\mathrm{UO}_{2} \mathrm{Cl}_{2}\right)$, uranyl fluoride $\left(\mathrm{UO}_{2} \mathrm{~F}_{2}\right)$, and uranium tetrafluoride $\left(\mathrm{UF}_{4}\right)$-were borrowed from a mineral repository stored at the Life Sciences Laboratory Complex at Pacific Northwest National Laboratory (PNNL). Sodium nitrate was obtained from Sigma-Aldrich (St. Louis, MO) and was included for comparison to uranium nitrate. For each sample, $\sim 5 \mathrm{mg}$ of powder was crushed onto a thin $(0.25 \mathrm{~mm}), 10 \mathrm{~mm}$ diameter indium foil disk, which was secured to a $10 \mathrm{~mm}$ diameter, $0.5 \mathrm{~mm}$ thick copper disk with double-sided conductive carbon tape. Sample disks were stored in high vacuum prior to packaging for shipment to the Synchrotron Radiation Center (SRC) in Stoughton, WI.

X-ray absorption spectra were collected at the HERMON beamline at the SRC, a bending magnet beamline equipped with a spherical grating monochromator, using the X-ray absorption end-station [27]. Sample disks were mounted on the sample arm using double-sided conductive carbon tape. Samples were placed in an airlock and pumped down to at least $3 \times 10^{-9}$ torr prior to transfer to the sample chamber. Once loaded, the samples were exposed to an incident beam with a slit-defined beam size of $20 \mu \mathrm{m}$. An X-ray probe of this size would encompass hundreds of particles, ensuring that the results would represent the bulk of the sample and not be dependent on the orientation of individual particles or grains. The oxygen K-edges were obtained for uranyl nitrate, uranyl chloride, uranyl fluoride, and sodium nitrate with the following scan parameters: 
$515 \mathrm{eV}$ to $528 \mathrm{eV}$ in $0.5 \mathrm{eV}$ steps, $528 \mathrm{eV}$ to $543 \mathrm{eV}$ in $0.1 \mathrm{eV}$ steps, $543 \mathrm{eV}$ to $550 \mathrm{eV}$ in $0.5 \mathrm{eV}$ steps, and $550 \mathrm{eV}$ to $580 \mathrm{eV}$ in $1 \mathrm{eV}$ steps. The fluorine $\mathrm{K}$-edges were obtained for uranyl fluoride and uranium tetrafluoride with the following parameters: $670 \mathrm{eV}$ to $682 \mathrm{eV}$ in $1 \mathrm{eV}$ steps, $682 \mathrm{eV}$ to $702 \mathrm{eV}$ in $0.1 \mathrm{eV}$ steps, $702 \mathrm{eV}$ to $710 \mathrm{eV}$ in $0.5 \mathrm{eV}$ steps, and $710 \mathrm{eV}$ to $740 \mathrm{eV}$ in $1 \mathrm{eV}$ steps. For both edges, dwell times were 1 second per data point. At least three sweeps were performed for each edge of each sample, and possibly more depending on the signal-to-noise level. The sample position was moved several hundred microns between sweeps in order to minimize the effects of beam damage. Spectra from individual sweeps were examined prior to averaging to confirm sample homogeneity.

X-ray absorption was detected through total electron yield (TEY), the technique of choice when samples are much thicker than the X-ray absorption length [28]. The TEY signal is directly proportional to the sample X-ray absorption coefficient so long as the absorption length of the X-ray probe is much longer than the electron escape depth, and as long as the experimental geometry does not place the $\mathrm{X}$-ray probe at grazing incidence [29-31]. For most of the materials presented in this work, the absorption length is roughly $200 \mathrm{~nm}$ right above the oxygen K-edge, whereas for most materials the mean free path for electrons in the soft X-ray regime is on the order of $10-100 \AA$ [32]. The X-ray absorption end-station at the SRC also offered total fluorescence yield (TFY) measurement capabilities; however, this detection mode was not used because the sample thickness would have introduced self-absorption artifacts. The signal from an upstream gold mesh served as a monitor for the beam intensity, $I_{0}$. Incident flux was on the order of $10^{8}$ photons per second. For a typical experiment, the TEY count rate 
immediately below the edge was $9 \times 10^{6}$ counts per second, while the count rate was $1 \times 10^{7}$ counts per second far above the absorption edge, yielding an edge jump of $\sim 1.1$. The ratio of the TEY and $\mathrm{I}_{0}$ signals plotted against $\mathrm{X}$-ray energy yielded an absorption edge. I0-normalized data were averaged for each sample and loaded into SIXPACK [33] for background subtraction and spectrum normalization. Edge jumps were normalized to 1.0. The pre-edge region was modeled as a line, and the post-edge as a quadratic spline with five knots.

X-ray powder diffraction (XRD) measurements were carried out for each uranium sample to confirm their purity. Sample disks were sealed between two pieces of Kapton tape and sent to the Environmental Molecular Sciences Laboratory (EMSL) at PNNL. Powder diffraction patterns were recorded using a Rigaku D/Max Rapid II micro diffraction system. X-rays were generated at $35 \mathrm{kV}$ and $25 \mathrm{~mA}$ from a rotating $\mathrm{Cr}$ target $(\lambda=2.2910 \AA)$ and focused into a parallel beam before passing through a $300 \mu \mathrm{m}$ diameter collimator onto the specimen. The incident beam direction was fixed at 25 degrees to the sample surface and the diffracted intensities recorded on a large 2D image plate. The $2 \mathrm{D}$ images were integrated between 25 and $150^{\circ} 2 \theta$ to give powder traces which were compared to previously-published reference patterns from the International Centre for Diffraction Data using JADE (version 9, Materials Data Inc., California) in order to make compound identifications. The reference patterns included various hydrated forms for each compound of interest (e.g., a hydrated form of uranyl fluoride, $\mathrm{UO}_{2} \mathrm{~F}_{2} \cdot 1.571 \mathrm{H}_{2} \mathrm{O}$, in addition to the anhydrous $\mathrm{UO}_{2} \mathrm{~F}_{2}$ structure).

Calculated spectra were generated in Quantum ESPRESSO using the XCH method [34]. Briefly, energy dependent transition amplitudes were calculated using 
density functional theory $[35,36]$. A plane wave basis set with periodic boundary conditions was used in conjunction with ultrasoft pseudopotentials [37] to model the valence electronic structure. The exchange-correlation energy was approximated via the Perdew-Burke-Ernzerhof [38] exchange-correlation functional within the generalized-gradient approximation. To simulate the experimental promotion of an oxygen or fluorine core electron to the valence states, the $\mathrm{XCH}$ calculations explicitly include both a full core hole (via a modified pseudopotential) and an extra electron in the lowest energy valence state. Self-consistent energies were calculated with the PWscf, part of the Quantum ESPRESSO package [39]. The calculated spectra were all uniformly broadened by Gaussian convolution of $0.2 \mathrm{eV}$ full width at half maximum. To help correct for known band gap underestimation in DFT, the energy axes for all calculated spectra were stretched by $20 \%$. The calculated spectra are aligned by reference to a theoretical standard, the isolated atom of the excited element, as described in England et al. [40]. This alignment scheme requires one empirical shift to align with experimental spectra. Structures for each calculation were taken from the published sources. The simulated structures include uranyl dinitrate trihydrate [41], uranyl nitrate hydroxide hydrate [42], anhydrous uranyl fluoride [43], uranyl fluoride hydrate [44], anhydrous uranyl chloride [45], uranyl chloride monohydrate [46], uranyl chloride trihydrate [47], and uranyl chloride hydroxide hydrate [48]. In most cases, hydrogen atoms were added to the structure and the unit cell and hydrogen positions relaxed prior to spectral calculations.

To simulate molecular motion inherently sampled in the experiment at room temperature, the relaxed structures were also given thermal energy and molecular 
motion allowed to propagate in time using the VASP program [49]. First-principles DFTbased molecular dynamics simulations were run at $300 \mathrm{~K}$. A molecular dynamics snapshot sampled 10 ps after equilibration was subsequently used to calculate the X-ray absorption spectrum as described above. Rather than averaging a few oxygen atoms across multiple snapshots, these spectra sample the same stochastic processes by averaging over the 40-100 oxygen atoms in the cell.

\section{Results and Discussion}

\subsection{Oxygen K-Edge Spectroscopy}

Many uranium compounds incorporate oxygen, including various uranium oxides and numerous minerals containing the uranyl $\left(\mathrm{UO}_{2}{ }^{2+}\right)$ ion. Therefore, scanning the oxygen $\mathrm{K}$-edge is a promising way to obtain chemical state information on a variety of uranium compounds of interest. However, not all of the oxygen present in the sample is bound directly to the uranium atom. For example, counterions in the sample can have significant oxygen content. Another potential complication to this work is the presence of water in the sample, since this could contribute intensity to the overall oxygen K-edge absorption. Under normal atmospheric conditions, bound water can represent a significant portion of a mineral's overall oxygen content. For example, in liebigite $\left(\mathrm{Ca}_{2}\left(\mathrm{UO}_{2}\right)\left(\mathrm{CO}_{3}\right)_{3} \bullet 11\left(\mathrm{H}_{2} \mathrm{O}\right)\right)$, water molecules contain half of the total oxygen content. Therefore, it was necessary to compare the experimentally-derived spectra to spectra calculated from published crystal structures, in order to determine the relative contributions of the various oxygen atom types to the overall absorption spectrum. 
Our XRD results suggested that the uranyl minerals examined here bind water under normal atmospheric conditions. Accordingly, the powder diffraction pattern from the $\mathrm{UO}_{2} \mathrm{~F}_{2}$ sample was most consistent with $\mathrm{UO}_{2} \mathrm{~F}_{2} \cdot 1.5\left(\mathrm{H}_{2} \mathrm{O}\right)$, the $\mathrm{UO}_{2} \mathrm{Cl}_{2}$ sample was found to actually be uranyl chloride hydroxide dihydrate, $\mathrm{UO}_{2}(\mathrm{OH}) \mathrm{Cl} \cdot 2\left(\mathrm{H}_{2} \mathrm{O}\right)$, and the $\mathrm{UO}_{2}\left(\mathrm{NO}_{3}\right)_{2}$ sample was found to be uranyl nitrate hydroxide dihydrate, $\mathrm{UO}_{2} \mathrm{NO}_{3} \mathrm{OH} \cdot 2\left(\mathrm{H}_{2} \mathrm{O}\right)$. However, it is unknown what the hydration state of these compounds would be under the high vacuum conditions present at a typical soft X-ray beamline, since presumably these conditions would remove much of the water content. Previously, our group examined the oxygen K-edge absorption of a cobalt chloride hexahydrate sample [23]. The sample color changed from purple to light blue under vacuum, indicating loss of inner-sphere water (i.e., water molecules directly bound to the metal center). However, we were able to detect a weak oxygen K-edge in the dehydrated sample, suggesting that at least some residual water was present. That same study found that soft X-ray scans of liebigite $\left(\mathrm{Ca}_{2}\left(\mathrm{UO}_{2}\right)\left(\mathrm{CO}_{3}\right)_{3} \bullet 11\left(\mathrm{H}_{2} \mathrm{O}\right)\right)$ and sodium uranyl carbonate $\left(\mathrm{Na}_{4}\left(\mathrm{UO}_{2}\right)\left(\mathrm{CO}_{3}\right)_{3} \cdot\left(\mathrm{H}_{2} \mathrm{O}\right)\right)$ yielded nearly identical oxygen $\mathrm{K}$ edge absorption profiles despite the vast nominal difference in the number of incorporated water molecules, suggesting that water can easily be lost under the vacuum conditions required for soft X-ray absorption spectroscopy. It is likely that some residual water is present under vacuum, but that it is a minor contributor to the overall oxygen K-edge absorption spectrum if the mineral under study has significant oxygen content.

Figure 1 shows the oxygen K-edge absorption spectra for the uranyl chloride, uranyl fluoride, uranyl nitrate, and sodium nitrate samples. A direct comparison of the 
nitrate samples is shown at the top. Previous work from our group and elsewhere in the literature found that uranyl minerals exhibit an intense pre-edge transition at $\sim 531.3 \mathrm{eV}$, which is due to an oxygen $1 \mathrm{~s}$ to uranyl $\pi^{*}$ transition on the uranyl $\left(\mathrm{UO}_{2}{ }^{2+}\right)$ moiety $[14$, 23]. Comparing uranyl silicates to a potassium silicate model and uranyl phosphates to a sodium phosphate model, we found that the silicate and phosphate counterions contributed very little absorption intensity in the pre-edge region, and although a sodium bicarbonate model did exhibit a pre-edge transition at $534.0 \mathrm{eV}$, this transition was wellresolved from the uranyl pre-edge peak at $531.3 \mathrm{eV}$. Therefore, for these minerals, the presence of a sharp pre-edge peak in the oxygen $\mathrm{K}$-edge spectrum serves as a specific marker for the presence of a uranyl ion. However, this is not the case for uranyl nitrate. Both the uranyl nitrate and the sodium nitrate spectra show three prominent peaks at ca. $532 \mathrm{eV}, 538 \mathrm{eV}$, and $542 \mathrm{eV}$. The peak at $532 \mathrm{eV}$ has previously been observed in nitrate solutions and was attributed to an $O 1 s 1 a_{1} \rightarrow 2 b_{1}{ }^{*}$ transition on the nitrate ion [50]. This direct overlap creates difficulty distinguishing uranyl nitrate from other nitrate compounds. However, it is notable that the pre-edge peak of the uranyl nitrate compound is broader than that of the sodium nitrate sample (FWHM of $2.2 \mathrm{eV}$ vs. $1.2 \mathrm{eV}$ ). Furthermore, the position of the uranyl nitrate pre-edge peak is at slightly lower energy than the sodium nitrate pre-edge peak (532.1 eV vs. $532.0 \mathrm{eV})$, closer to the energy of a typical uranyl pre-edge transition. These facts suggest the uranyl pre-edge peak is present, but unresolved from the nitrate pre-edge peak in this sample. 


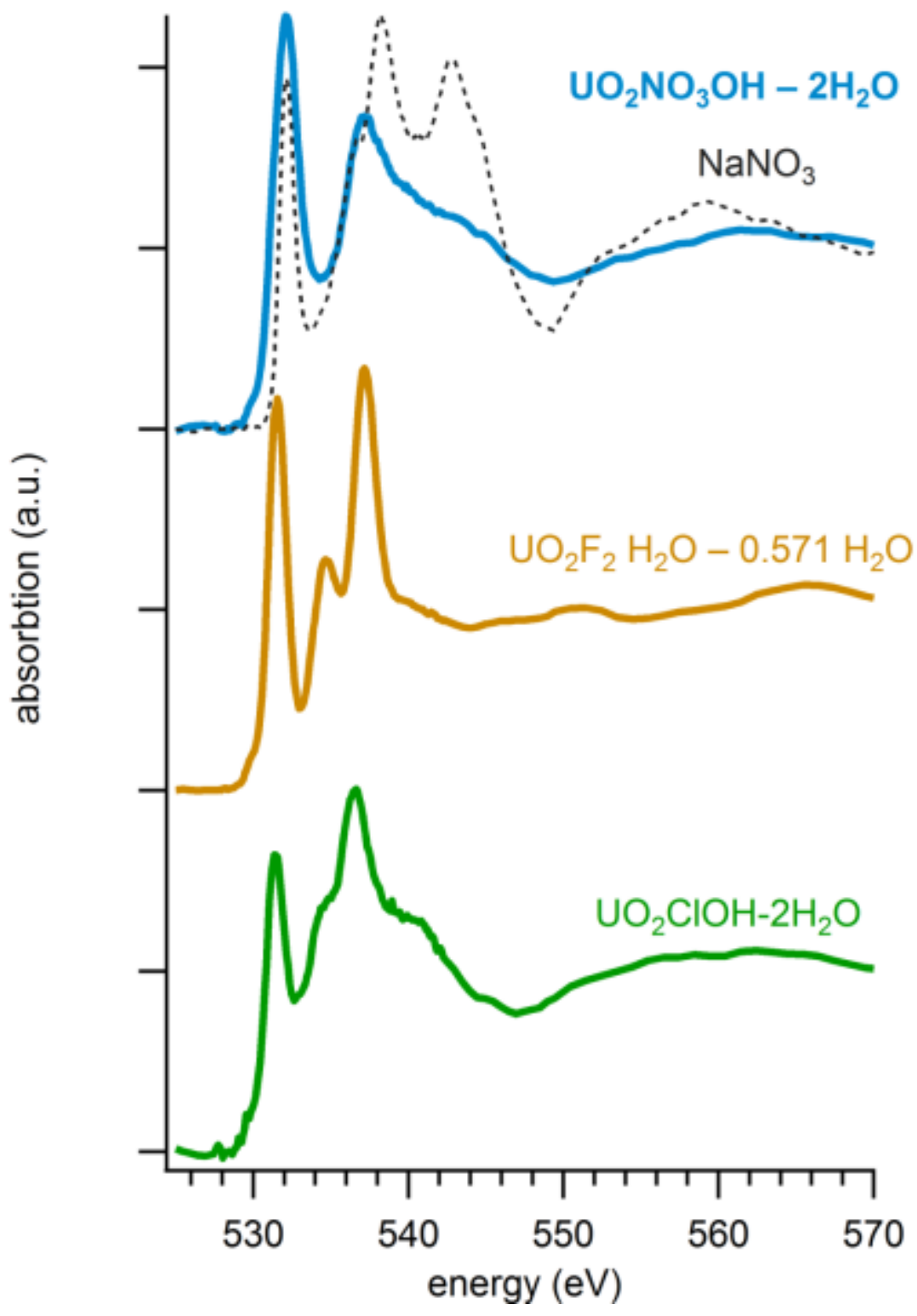

Figure 1. Comparison of the oxygen K-edge absorption spectra of the uranyl nitrate (blue solid line), sodium nitrate (black dashed line), uranyl fluoride (orange solid line), and uranyl chloride (green solid line) complexes. 
As a result of the spectral overlap, the task of compound identification and classification is more complicated for uranyl nitrate, since in a sample with significant background nitrate content, the presence of the pre-edge peak at $\sim 531.3 \mathrm{eV}$ will no longer be an unambiguous marker for the presence of uranyl. Although the overall absorption profile for uranyl nitrate is distinct from the sodium nitrate model, it is likely insufficient to distinguish uranyl nitrate from other nitrates that may be present in the background based on the oxygen K-edge absorption spectrum alone. In this instance, the specific presence of uranium can be confirmed by scanning the uranium N4,5 peaks at $736.2 \mathrm{eV}$ and $778.3 \mathrm{eV}$, which would be readily accessible by most soft X-ray beamlines. This step would be advisable in any event, in order to distinguish uranyl minerals from similar heavy metal minerals (e.g., minerals containing the plutonyl ion, $\left.\mathrm{PuO}_{2}{ }^{2+}\right)$.

The uranyl halide samples are compared in the middle and bottom of Figure 1. The uranyl fluoride scan shows three peaks-a sharp, intense pre-edge at $531.5 \mathrm{eV}$, a less intense peak at $534.5 \mathrm{eV}$, and a sharp, intense main-edge peak at $537.1 \mathrm{eV}$. Oxygen K-edge scans of $\mathrm{UO}_{2}(\mathrm{py})_{3} \mathrm{I}_{2}[14]$ show similar features, suggesting that the absorption intensity of $\mathrm{UO}_{2} \mathrm{~F}_{2}$ is due primarily to the uranyl unit, as expected. In contrast, the uranyl chloride spectrum exhibits four recognizable peaks-the uranyl pre-edge at $531.4 \mathrm{eV}$, a shoulder at $534.5 \mathrm{eV}$, a main-edge peak at $536.6 \mathrm{eV}$, and a low-intensity peak at $540.8 \mathrm{eV}$. Overall, the spectral features of the uranyl halide compounds are sufficient to be useful for compound identification as they are distinguishable from many of the common uranyl minerals [23]. 
In order to get a more detailed picture of the various spectral contributions to the oxygen K-edge absorption spectra, simulated spectra were calculated for $\mathrm{UO}_{2} \mathrm{NO}_{3} \mathrm{OH} \cdot 2\left(\mathrm{H}_{2} \mathrm{O}\right), \mathrm{UO}_{2} \mathrm{~F}_{2} \cdot 1.5\left(\mathrm{H}_{2} \mathrm{O}\right)$, and $\mathrm{UO}_{2} \mathrm{OHCl} \cdot 2\left(\mathrm{H}_{2} \mathrm{O}\right)$ using Quantum ESPRESSO. The results are shown in Figure 2. The calculated spectra (shown in black) reproduce the main features of the experimental spectra, adding validity to additional spectral interpretation via the calculations. In that vein, an additional advantage of the calculated spectra is the ability to attribute spectral contributions to the various oxygen atoms within the chemical system. Spectral contributions of chemical subclass, scaled by their relative atom count, are also shown in Figure 2. In general, the calculations shown in Figure 2 confirm that the uranyl moiety is responsible for the three spectral features discussed above, namely peaks at $\sim 532 \mathrm{eV}, \sim 534 \mathrm{eV}$, and $\sim 537 \mathrm{eV}$, and that additional water/hydroxide contribute mainly in the 535-540 eV range. 


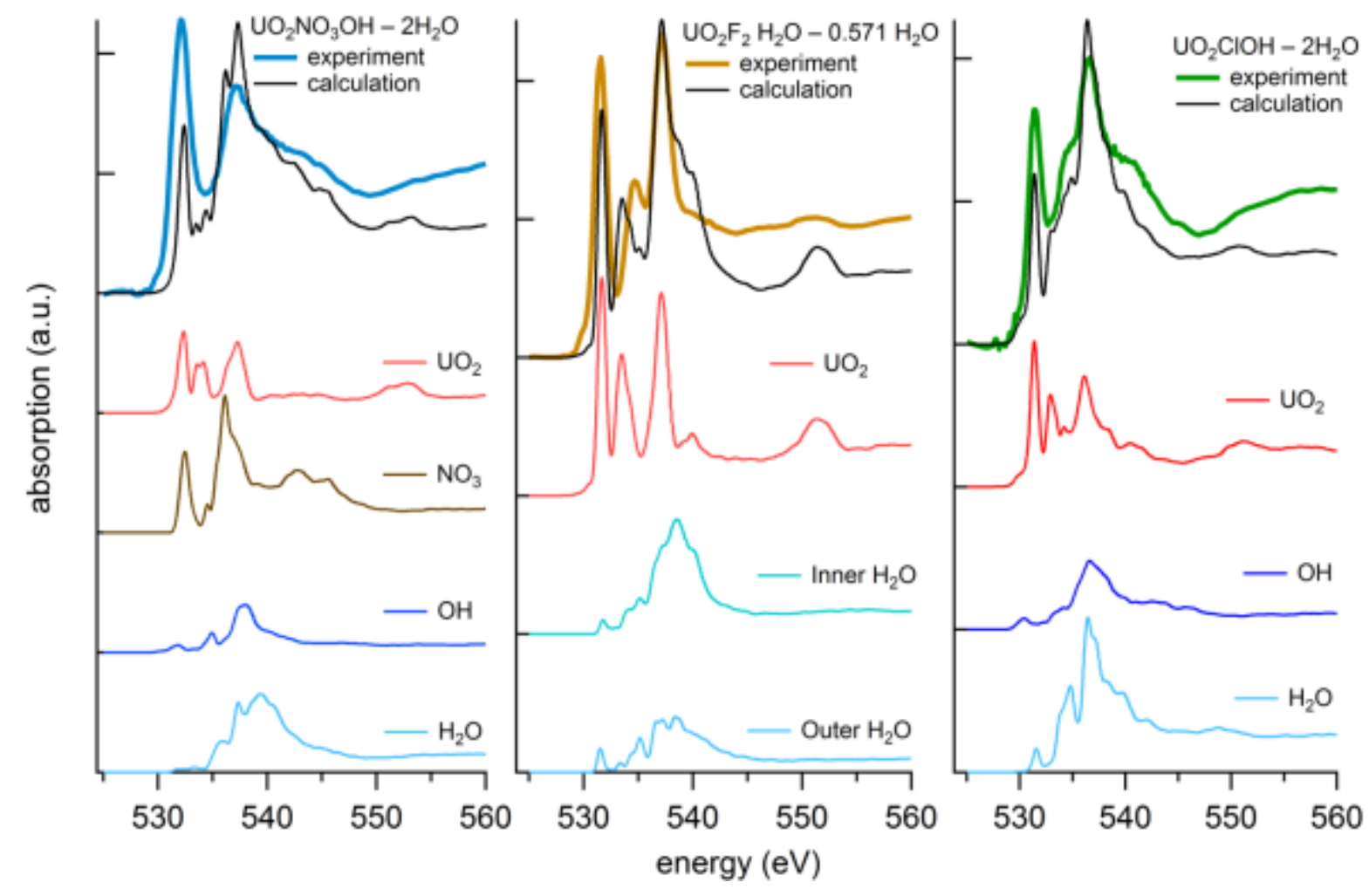

Figure 2. Comparison between experimental and calculated oxygen K-edge absorption spectra for the uranyl nitrate (left), uranyl fluoride (center), and uranyl chloride (right) complexes. The contributions from the various molecular components-uranyl ions, nitrate ions, hydroxide ions, and inner-sphere water (water directly bound to uranium) and outer-sphere water (water present in the sample, but not bound to uranium)-are shown below.

More specifically, the calculated uranyl nitrate spectrum (left column of Figure 2) matches the two prominent experimental peaks at $\sim 532 \mathrm{eV}$ and $\sim 537 \mathrm{eV}$, and a highenergy shoulder on the main-edge peak. However, the relative intensity of the two mains peaks does not match experiment. The calculated contribution from the uranyl ion (red spectrum) indicates that uranyl will contribute three peaks to the oxygen K-edge 
absorption spectrum at $\sim 532 \mathrm{eV}, \sim 534 \mathrm{eV}$, and $\sim 537 \mathrm{eV}$, consistent with previous experimental results [14]. The calculated spectrum from the nitrate ions within uranyl nitrate (shown in brown) confirms that this unit will add absorption intensity similar to the sodium nitrate spectrum. Since both the uranyl and the nitrate ions contribute absorption intensity at $\sim 532 \mathrm{eV}$ and $\sim 537 \mathrm{eV}$, the experimental (and calculated) peaks at these positions are relatively intense and broad, overwhelming the smaller uranyl peak at $534 \mathrm{eV}$. It is interesting to note that the main edge of the nitrate ion contributes most intensely at $\sim 536 \mathrm{eV}$ while the uranyl oxygens contribute main edge intensity centered at $\sim 537 \mathrm{eV}$. A higher resolution experimental spectrum would reveal the intermediate energy uranyl peak and better separate the uranyl and nitrate contributions to the main edge. The nitrate ion is also expected to absorb broadly at $542 \mathrm{eV}$. This peak, along with contributions from water/hydroxide, is the main source of spectral intensity at high energies (538-548 eV).

The breakdown of spectral contribution shown for uranyl nitrate in Figure 2 indicates that the relative intensity of the two main peaks (532 and $537 \mathrm{eV}$ ) could be influenced by the water content of the sample. Consequently, calculations were also carried out for the uranyl nitrate, uranyl fluoride, and uranyl chloride complexes under various hydration states in order to learn more about how the presence of water might affect the observed absorption spectra. The hydration states selected are ones that other groups have observed experimentally—see the Experimental section above for the specific compounds calculated. The results are shown in Figure 3. In addition, Figure 3 also shows the calculated spectra derived directly from the literature-obtained crystal structure and calculated spectra derived after molecular dynamics simulations of 
the same crystal structures. The left-hand side of Figure 3 repeats the experimental uranyl nitrate spectrum, along with calculated spectra for $\mathrm{UO}_{2} \mathrm{NO}_{3} \mathrm{OH} \cdot 2\left(\mathrm{H}_{2} \mathrm{O}\right)$ and $\mathrm{UO}_{2}\left(\mathrm{NO}_{3}\right)_{2} \cdot 3\left(\mathrm{H}_{2} \mathrm{O}\right)$. (The $\mathrm{UO}_{2} \mathrm{NO}_{3} \mathrm{OH} \cdot 2\left(\mathrm{H}_{2} \mathrm{O}\right)$ snapshot spectrum was presented in greater detail in Figure 2.) Both calculated spectra are qualitatively similar to the experimental nitrate complex, although the peak at $542 \mathrm{eV}$ (and $537 \mathrm{eV}$ ) is less pronounced in the uranyl nitrate hydroxide, consistent with the reduced uranyl to nitrate and uranyl to water ratios in this structure. It is interesting to note that the $\mathrm{UO}_{2} \mathrm{NO}_{3} \mathrm{OH} \cdot 2\left(\mathrm{H}_{2} \mathrm{O}\right)$ spectra calculated from the published crystal structure [42] and the spectra calculated following the molecular dynamics simulation reverse the relative intensity of the pre-edge and main edge peaks, with the spectrum calculated from the crystal structure reference providing a better match to the experimental intensities. Assuming this calculated spectral change is a true representation of the spectral changes with increased molecular motion, it is possible that the vacuum conditions under which the experimental uranyl nitrate spectrum was collected reduced the water content below the nominal stoichiometry, which would explain the relatively more prominent pre-edge peak observed in the experiment. 

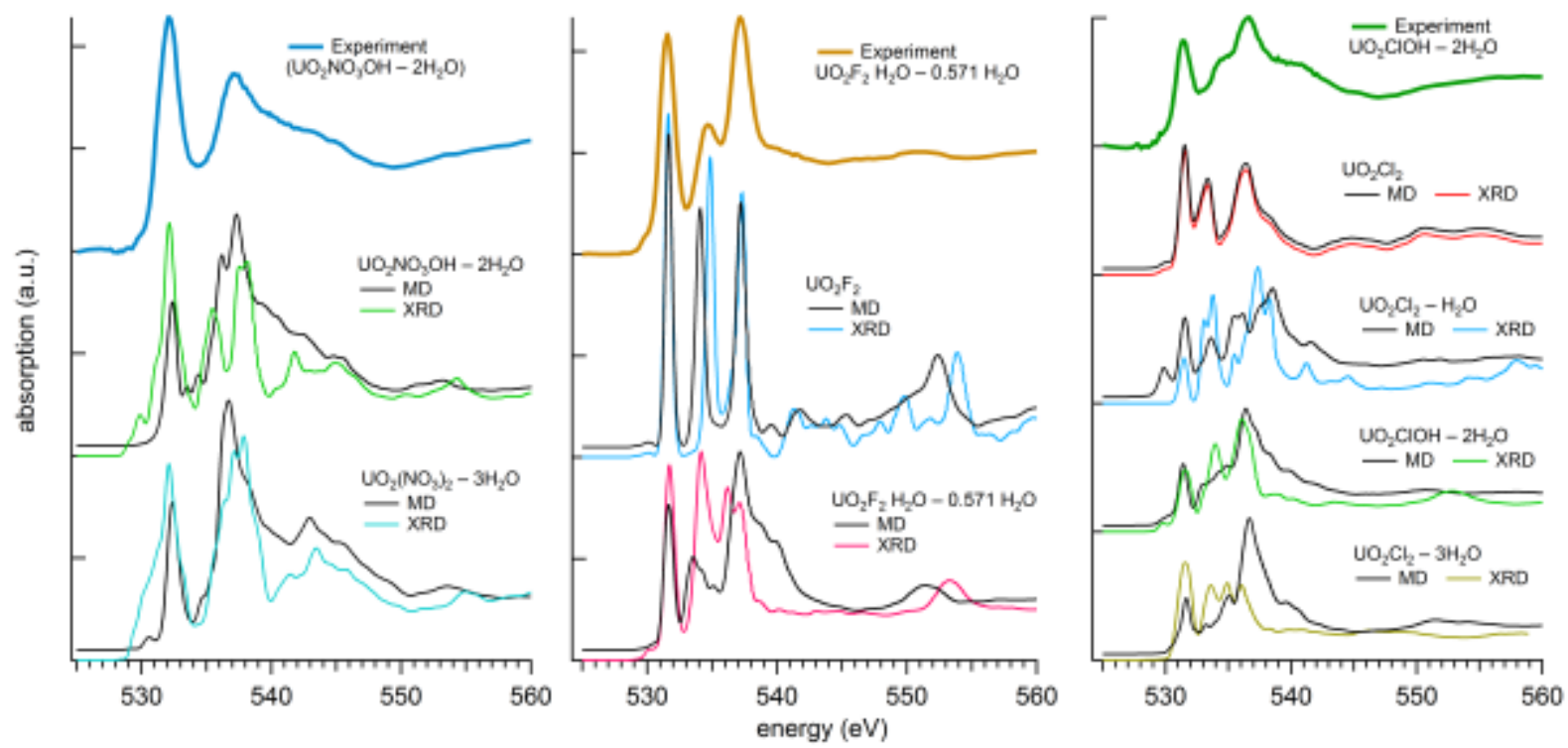

Figure 3. Comparison between the experimental uranyl nitrate (left), uranyl fluoride (center), and uranyl chloride (right) spectra with calculated spectra from various different hydration states. Spectra labeled "XRD" were calculated directly from experimentally available crystallography derived structure files, while spectra labeled "MD" were calculated after molecular dynamics simulations.

Analysis of the uranyl fluoride and uranyl chloride spectra and calculations (Figures 2 and 3) proceed similarly to that presented above for uranyl nitrate. The experimental and calculated uranyl fluoride spectra (middle of Figure 2) show three prominent peaks. The calculations show that these peaks are attributed to the uranyl ion (red spectrum). Both inner-sphere and outer-sphere water (i.e., water present in the sample, but not directly bound to a metal center) would be expected to contribute a broad peak around $539 \mathrm{eV}$ (trace shown in light blue), creating a high-energy shoulder off the third uranyl peak. This shoulder is not prominent in the experimental spectrum, again suggesting that water was lost under high-vacuum conditions. 
The calculated uranyl chloride spectrum (right side of Figure 2) is in excellent agreement with the experimental spectrum. Figure 3 also shows a wider range of uranyl chloride hydration state calculations, $\mathrm{UO}_{2} \mathrm{Cl}_{2}, \mathrm{UO}_{2} \mathrm{Cl}_{2} \cdot \mathrm{H}_{2} \mathrm{O}, \mathrm{UO}_{2} \mathrm{ClOH} \cdot 2\left(\mathrm{H}_{2} \mathrm{O}\right)$, and $\mathrm{UO}_{2} \mathrm{Cl}_{2} \cdot 3\left(\mathrm{H}_{2} \mathrm{O}\right)$. Of the calculated uranyl chloride spectra, the uranyl chloride hydroxide dihydrate complex, $\mathrm{UO}_{2} \mathrm{ClOH} \cdot 2\left(\mathrm{H}_{2} \mathrm{O}\right)$, models the experimental spectrum most closely. This result is consistent with the X-ray diffraction measurement results. It is unusual that a calculated spectrum derived from a doubly-hydrated structure should be such a good fit for the experimental spectrum, since in other samples there is evidence for water loss under vacuum conditions. However, it should be noted that the uranyl chloride hydroxide dehydrate complex is the only uranyl chloride complex to incorporate a hydroxide ion, and Figure 2 indicates that the presence of a hydroxide ion would make similar spectral contributions to the overall oxygen K-edge absorption as an incorporated water molecule. It is interesting to note the clear spectral differences between the calculated uranyl chloride structures. That is, based only on the calculated oxygen K-edge NEXAFS spectra, each of the four hydration states shown are clearly identifiable.

The calculations shown in Figure 3 highlight the potential sensitivity of oxygen Kedge spectra to hydration. While the experimental spectra were collected at an unknown hydration state attained under vacuum conditions, the calculations indicate that it is nominally the same state measured with XRD. The stark difference in the calculated spectra seen between different hydration states indicate that different hydrated forms of these anthropogenic compounds will show distinct spectral signatures. This opens the potential to probe chemical aging and weathering of uranyl 
compounds using a soft X-ray probe. For example, the reaction of dry uranyl chloride with humid air may proceed through various hydration states and the calculations indicate that the kinetics of these reactions could be followed with oxygen K-edge NEXAFS.

\subsection{Fluorine K-Edge Spectroscopy}

Fluorinated uranium compounds are not as common as oxygenated uranium compounds; however, these compounds are crucial to the nuclear fuel cycle. The fluorine $\mathrm{K}$-edge is close in energy to the oxygen $\mathrm{K}$-edge, and would be accessible to most soft X-ray beamlines. In addition, interpretation of the fluorine K-edge would not be complicated by the possible presence of residual water. Therefore, it would be useful to examine the spectral features of fluorinated uranium compounds and compare these to the fluorine K-edges of potential "background" fluorinated compounds.

The experimental fluorine K-edge absorption spectra for $\mathrm{UO}_{2} \mathrm{~F}_{2}$ and $\mathrm{UF}_{4}$ are shown in Figure 4, along with spectra (taken from the literature) for fluorite [51], fluorapatite [52], Teflon [53], and lithium fluoride [54]. The fluorine near-edge for $\mathrm{UO}_{2} \mathrm{~F}_{2}$ has two main peaks - a medium-intensity pre-edge at $687.4 \mathrm{eV}$, and a sharp, intense main-edge peak at $692.6 \mathrm{eV}$. In contrast, the $\mathrm{UF}_{4}$ main-edge peak is at $693.3 \mathrm{eV}$, while there is an unresolved shoulder on the low-energy side at $\sim 690.0 \mathrm{eV}$. Figure 4 indicates that the fluorine $\mathrm{K}$-edge spectra for both $\mathrm{UO}_{2} \mathrm{~F}_{2}$ and $\mathrm{UF}_{4}$ are significantly different from the fluorine K-edge spectra from fluorite, fluorapatite, Teflon, and lithium fluoride. Fluorite and fluorapatite are two common fluorine-containing minerals and would represent much of the "natural" fluorine background. Teflon and lithium fluoride 
represent common anthropogenic sources of fluorine which may be present in a laboratory setting. Teflon is commonly used in non-stick coatings and tapes, while lithium fluoride sees multiple uses, including thermoluminescent radiation detectors [55] and UV filter windows [56]. $\mathrm{UO}_{2} \mathrm{~F}_{2}$ and $\mathrm{UF}_{4}$ can be readily distinguished from these more common fluorine compounds on the basis of their fluorine K-edge spectra alone. 


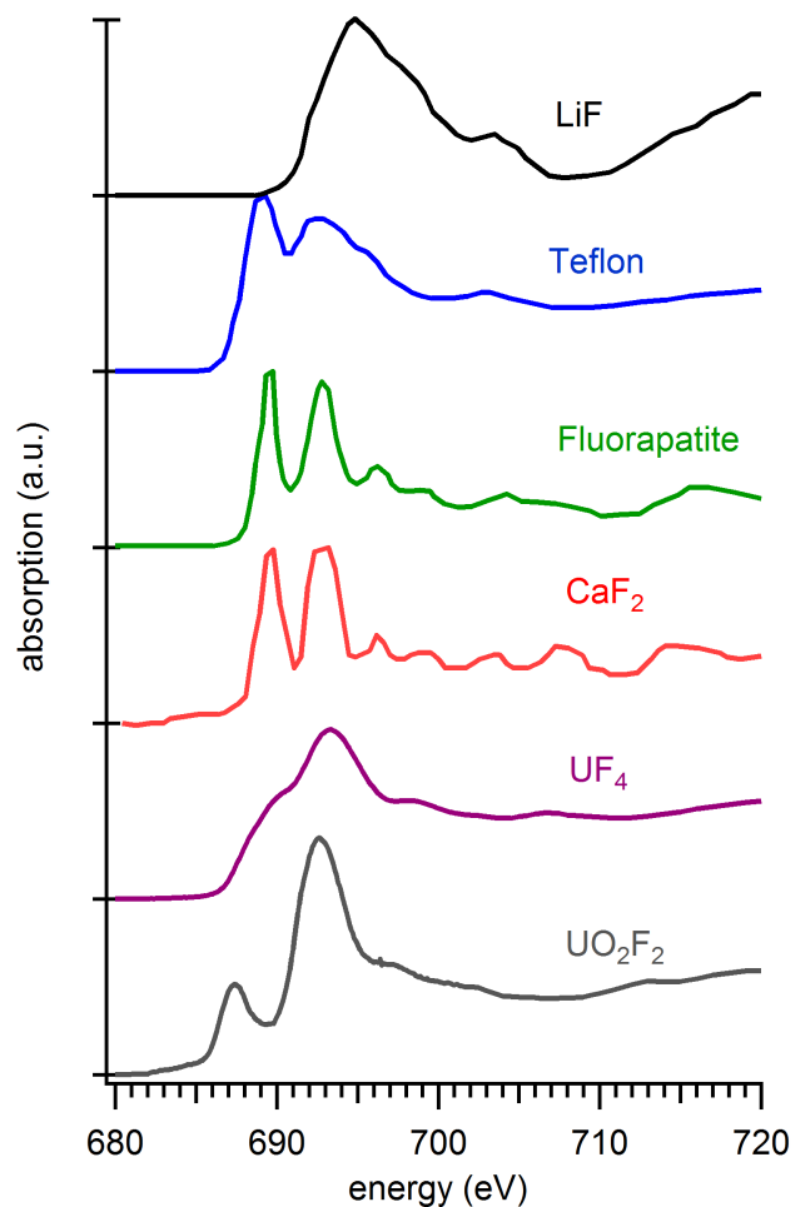

Figure 4. Comparison between the fluorine K-edge absorption spectra of uranyl fluoride (grey), uranium tetrafluoride (purple), calcium fluoride (red), fluorapatite (green), Teflon (blue), and lithium fluoride (black). The calcium fluoride, fluorapatite, Teflon, and lithium fluoride spectra were taken from the literature (references shown in Section 3). The fluoridated uranium compounds show fluorine K-edge absorption profiles that are clearly distinct from various other sources of "background" fluorine. 
Fluorine K-edge $\mathrm{X}$-ray absorption spectra were calculated for $\mathrm{UO}_{2} \mathrm{~F}_{2}$ and $\mathrm{UO}_{2} \mathrm{~F}_{2} \cdot 1.5\left(\mathrm{H}_{2} \mathrm{O}\right)$ in order to assess the effect of hydration state on the absorption profiles. The results are shown in Figure 5. The calculated spectra are excellent matches to the experimental absorption spectrum, with more structure seen in the $\mathrm{UO}_{2} \mathrm{~F}_{2}$ spectra. The calculated spectra were numerically broadened so as to retain spectral detail rather than to empirically match experiment. With empirical broadening, either $\mathrm{UO}_{2} \mathrm{~F}_{2}$ or the $\mathrm{UO}_{2} \mathrm{~F}_{2} \cdot 1.5\left(\mathrm{H}_{2} \mathrm{O}\right)$ would be an excellent match to experiment. These results demonstrate how robust the fluorine K-edge absorption spectra are with respect to hydration state, in contrast to the oxygen K-edge absorption spectra. This insensitivity to hydration state makes the fluorine $\mathrm{K}$-edge a powerful complement to the oxygen $\mathrm{K}$ edge absorption data. 


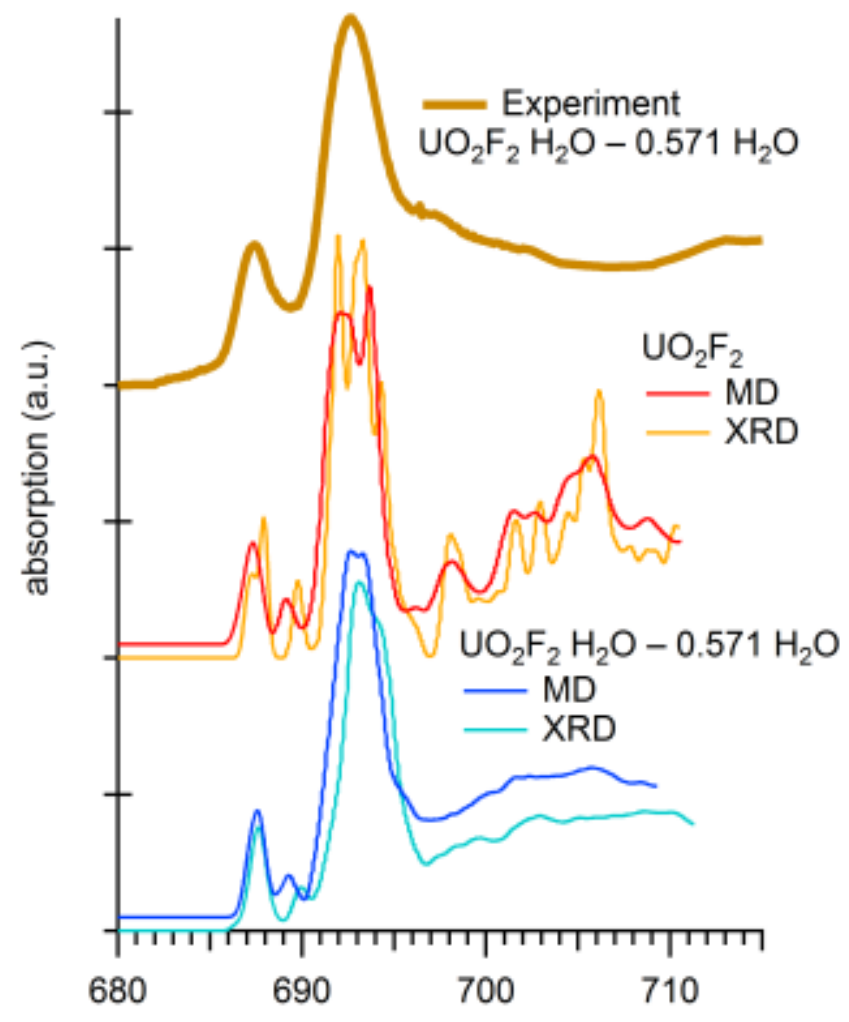

Figure 5. Comparison between the experimental fluorine K-edge absorption spectrum (brown) and calculated spectra for dry uranyl fluoride (red and orange) and the hydrated complex (dark and light blue). Spectra labeled "XRD" were calculated directly from experimentally available structure files, while spectra labeled "MD" were calculated after molecular dynamics simulations. The presence of water adds spectral intensity to the post-peak region 


\section{Conclusions}

We have found in general that uranyl compounds under vacuum will lose at least some of their water content; therefore, the relative contribution of water to the overall oxygen K-edge absorption will be somewhat less than the nominal structure of the compound would indicate. Furthermore, in cases where the data do suggest significant water content even under vacuum (e.g., the uranyl chloride dioxide dihydrate complex), the water molecules primarily add intensity to the main edge at $535-540 \mathrm{eV}$, while exhibiting no intensity in the pre-edge region. Thus, while the ratio of the intensities of the pre-edge peak and main-edge peak will shift in the presence of water, the pre-edge peak at $531.3 \mathrm{eV}$ can still be used to identify uranyl compounds. One potential complication is the presence of a similar pre-edge peak in the nitrate molecule at $532 \mathrm{eV}$. This peak can probably not be resolved from the uranyl pre-edge under normal conditions; therefore, other spectral markers will have to be used in the event of a sample with high nitrate content. The uranium N4,5 edges will be useful to confirm the specific presence of uranium.

The fluorine K-edge would not suffer any complications due to hydration state, as confirmed by the spectral simulations presented here. Furthermore, fluorinated uranium compounds are of particular interest to the nuclear forensics community. The fluorine Kedge data presented here demonstrate that the spectra derived from $\mathrm{UF}_{4}$ and $\mathrm{UO}_{2} \mathrm{~F}_{2}$ are distinct from spectra derived from several potentially significant natural and manmade background sources of fluorine. Simulated spectra demonstrate that the fluorine K-edge is minimally perturbed by extraneous water. 
In principle, the bulk oxygen K-edge and fluorine K-edge spectra presented here can be applied to identify spectra obtained from spatially-resolved techniques such as EELS or STXM. This would be useful for chemically identifying uranium-bearing submicron particles of interest against a potentially inhomogeneous background.

\section{Acknowledgements}

The research described in this paper is part of the Chemical Imaging Initiative at Pacific Northwest National Laboratory. It was conducted under the Laboratory Directed Research and Development Program at PNNL, a multiprogram national laboratory operated by Battelle for the U.S. Department of Energy. XRD measurements were conducted at the Environmental Molecular Sciences Laboratory, a DOE Office of

Science User Facility sponsored by the Office of Biological and Environmental Research and located at PNNL. Computational resources were provided by The Molecular Foundry at Lawrence Berkeley National Laboratory. The authors would like to thank John Fulton at PNNL for reviewing this document.

\section{References}

1. Finch, R.J. and R.C. Ewing, The corrosion of uraninite under oxidizing conditions. Journal of Nuclear Materials, 1992. 190: p. 133-156.

2. Maher, K., J.R. Bargar, and G.E. Brown Jr, Environmental speciation of actinides. Inorganic chemistry, 2012. 52(7): p. 3510-3532.

3. Spedding, F.H., H.A. Wilhelm, and W. Keller, The Production of Uranium by the Reduction of UF4 by $M g, 1945$, DTIC Document.

4. Uhlír, J., Chemistry and technology of Molten Salt Reactors - history and perspectives. Journal of Nuclear Materials, 2007. 360(1): p. 6-11.

5. Croff, A., et al., Assessment of preferred depleted uranium disposal forms. ORNL/TM-2000/161, Oak Ridge National Laboratory, Oak Ridge, Tenn., June, 2000.

6. Carter, J.A. and D.M. Hembree, Formation and characterization of UO2F2 particles as a result of UF6 hydrolysis. 1998: Oak Ridge National Laboratory. 
7. Kips, R., et al., Determination of fluorine in uranium oxyfluoride particles as an indicator of particle age. Spectrochimica Acta Part B: Atomic Spectroscopy, 2009. 64(3): p. 199-207.

8. Wagner, G.L., et al., Morphologic and Chemical Characterization of Products from Hydrolysis of UF6. Journal of Fluorine Chemistry, (0).

9. Wang, Z., et al., Fluorescence spectroscopy of U (VI)-silicates and U (VI)-contaminated Hanford sediment. Geochimica et cosmochimica acta, 2005. 69(6): p. 1391-1403.

10. Bernhard, G., et al., Uranium speciation in waters of different uranium mining areas. Journal of Alloys and Compounds, 1998. 271: p. 201-205.

11. Pointurier, F. and O. Marie, Identification of the chemical forms of uranium compounds in micrometer-size particles by means of micro-Raman spectrometry and scanning electron microscope. Spectrochimica Acta Part B: Atomic Spectroscopy, 2010. 65(9): p. 797-804.

12. Stefaniak, E.A., et al., New insight into $\mathrm{UO}_{2} \mathrm{~F}_{2}$ particulate structure by micro-Raman spectroscopy. Journal of Molecular Structure, 2013. 1040: p. 206-212.

13. de Groot, F. and A. Kotani, Core level spectroscopy of solids. 2008: CRC press.

14. Fillaux, C., et al., Investigating the electronic structure and bonding in uranyl compounds by combining NEXAFS spectroscopy and quantum chemistry. Physical Chemistry Chemical Physics, 2010. 12(42): p. 14253-14262.

15. Odelius, M., Molecular dynamics simulations of fine structure in oxygen K-edge $x$-ray emission spectra of liquid water and ice. Physical Review B, 2009. 79(14): p. 144204.

16. Mann, K., Near-Edge X-ray Absorption Fine Structure Measurements Using a Laboratory-Scale XUV Source. Short Wavelength Laboratory Sources, 2014: p. 407.

17. Sedlmair, J., et al., Characterization of refractory organic substances by NEXAFS using a compact $X$-ray source. Journal of Soils and Sediments, 2012. 12(1): p. 24-34.

18. Peth, C., F. Barkusky, and K. Mann, Near-edge $x$-ray absorption fine structure measurements using a laboratory-scale XUV source. Journal of Physics D: Applied Physics, 2008. 41(10): p. 105202.

19. Seidler, G.T., et al., A Modern Laboratory XAFS Cookbook. Journal of Physics: Conference Series, 2016. 712(1): p. 012015.

20. Haensel, R., P. Rabe, and B. Sonntag, Optical absorption of cerium, cerium oxide, praseodymium, praseodymium oxide, neodymium, neodymium oxide and samarium in the extreme ultraviolet. Solid State Communications, 1970. 8(22): p. 1845-1848.

21. Copping, R., et al., Toward Equatorial Planarity about Uranyl: Synthesis and Structure of Tridentate Nitrogen-Donor $\{\mathrm{UO}\}\}(2+)$ Complexes. Inorganic Chemistry, 2014. 53(5): p. 25062515.

22. Conradson, S.D., et al., Possible Bose-condensate behavior in a quantum phase originating in a collective excitation in the chemically and optically doped Mott-Hubbard system $\cup_{2+x}$. Physical Review B, 2013. 88(11): p. 115135.

23. Ward, J.D., et al., Identification of Uranyl Minerals Using Oxygen K-Edge X-Ray Absorption Spectroscopy. Geostandards and Geoanalytical Research, 2015: p. 1-14.

24. Higgins, I., et al., The Excer Process. Preparing Uranium Tetrafluoride by lon Exchange and Electrolysis. Industrial \& Engineering Chemistry, 1958. 50(3): p. 285-292.

25. Sibbens, G., A. Moens, and R. Eykens, Preparation and sublimation of uranium tetrafluoride for the production of thin 235UF4 targets. Journal of Radioanalytical and Nuclear Chemistry, 2015: p. 1-4.

26. Abe, Y., et al., Detection of Uranium and Chemical State Analysis of Individual Radioactive Microparticles Emitted from the Fukushima Nuclear Accident Using Multiple Synchrotron Radiation X-ray Analyses. Analytical chemistry, 2014. 86(17): p. 8521-8525. 
27. Bissen, M., et al., First results of SRC's new high-energy resolution variable line density grating monochromator beamline: HERMON. Review of scientific instruments, 1995. 66(2): p. 20722074.

28. Bunker, G., Introduction to XAFS: a practical guide to X-ray absorption fine structure spectroscopy. 2010: Cambridge University Press.

29. Stöhr, J., NEXAFS spectroscopy. Vol. 25. 1992: Springer.

30. Nakajima, R., J. Stöhr, and Y. Idzerda, Electron-yield saturation effects in L-edge x-ray magnetic circular dichroism spectra of Fe, Co, and Ni. Physical Review B, 1999. 59(9): p. 6421.

31. Abbate, M., et al., Probing depth of soft $x$-ray absorption spectroscopy measured in total-electron-yield mode. Surface and Interface Analysis, 1992. 18(1): p. 65-69.

32. Powell, C. and A. Jablonski, Evaluation of electron inelastic mean free paths for selected elements and compounds. Surface and interface analysis, 2000. 29(2): p. 108-114.

33. Webb, S., SIXpack: a graphical user interface for XAS analysis using IFEFFIT. Physica Scripta, 2005. 2005(T115): p. 1011.

34. Prendergast, D. and G. Galli, X-ray absorption spectra of water from first principles calculations. Physical Review Letters, 2006. 96(21).

35. Hohenberg, P. and W. Kohn, INHOMOGENEOUS ELECTRON GAS. Physical Review B, 1964. 136(3B): p. B864-+.

36. Kohn, W. and L.J. Sham, SELF-CONSISTENT EQUATIONS INCLUDING EXCHANGE AND CORRELATION EFFECTS. Physical Review, 1965. 140(4A): p. 1133-\&.

37. Vanderbilt, D., Soft self-consistent pseudopotentials in a generalized eigenvalue formalism. Physical Review B, 1990. 41(11): p. 7892-7895.

38. Perdew, J.P., K. Burke, and M. Ernzerhof, Generalized gradient approximation made simple. Physical Review Letters, 1996. 77(18): p. 3865-3868.

39. Paolo, G., et al., QUANTUM ESPRESSO: a modular and open-source software project for quantum simulations of materials. Journal of Physics: Condensed Matter, 2009. 21(39): p. 395502.

40. England, A.H., et al., On the hydration and hydrolysis of carbon dioxide. Chemical Physics Letters, 2011. 514(4-6): p. 187-195.

41. Hughes, K.-A. and P.C. Burns, Uranyl dinitrate trihydrate, UO2 (NO3) 2 (H2O) 3. Acta Crystallographica Section C: Crystal Structure Communications, 2003. 59(1): p. i7-i8.

42. Perrin, A., Structure cristalline du nitrate de dihydroxo diuranyle tetrahydrate. Acta Crystallographica Section B, 1976. 32(6): p. 1658-1661.

43. Atoji, M. and M. McDermott, The crystal structure of anhydrous UO2F2. Acta Crystallographica Section B: Structural Crystallography and Crystal Chemistry, 1970. 26(10): p. 1540-1544.

44. Mikhajlov, Y.N., et al., Synthesis and structure of new modification of monoaquadifluorouranyl hydrate. Russian Journal of Inorganic Chemistry, 2002. 47(12): p. 1821-1826.

45. Taylor, J. and P. Wilson, The structure of anhydrous uranyl chloride by powder neutron diffraction. Acta Crystallographica Section B: Structural Crystallography and Crystal Chemistry, 1973. 29(5): p. 1073-1076.

46. Taylor, J. and P. Wilson, The structure of uranyl chloride monohydrate by neutron diffraction and the disorder of the water molecule. Acta Crystallographica Section B: Structural Crystallography and Crystal Chemistry, 1974. 30(1): p. 169-175.

47. Debets, P., The structures of uranyl chloride and its hydrates. Acta Crystallographica Section B: Structural Crystallography and Crystal Chemistry, 1968. 24(3): p. 400-402.

48. Aberg, M., The Crystal Structure of [(UO2) $2(\mathrm{OH}) 2 \mathrm{Cl} 2$ (H2O) 4]. Acta chem. scand, 1969. 23(3): p. 6.

49. Kresse, G. and J. Furthmüller, Efficient iterative schemes for ab initio total-energy calculations using a plane-wave basis set. Physical review B, 1996. 54(16): p. 11169. 
50. Křepelová, A., et al., The nature of nitrate at the ice surface studied by XPS and NEXAFS. Physical Chemistry Chemical Physics, 2010. 12(31): p. 8870-8880.

51. Kurmaev, E., et al., Observation of fluorapatite formation under hydrolysis of tetracalcium phosphate in the presence of KF by means of soft X-ray emission and absorption spectroscopy. Journal of Materials Science: Materials in Medicine, 2002. 13(1): p. 33-36.

52. Kanno, C.M., et al., Novel apatite-based sorbent for defluoridation: synthesis and sorption characteristics of nano-micro-crystalline hydroxyapatite-coated-limestone. Environmental science \& technology, 2014. 48(10): p. 5798-5807.

53. Okudaira, K.K., et al., Photodegradation of poly (tetrafluoroethylene) and poly (vinylidene fluoride) thin films by inner shell excitation. Surface Review and Letters, 2002. 9(01): p. 335-340.

54. Hämäläinen, K., et al., Momentum dependence of fluorine K-edge core exciton in LiF. Physical Review B, 2002. 65(15): p. 155111.

55. Seco, J., B. Clasie, and M. Partridge, Review on the characteristics of radiation detectors for dosimetry and imaging. Physics in medicine and biology, 2014. 59(20): p. R303.

56. Symonds, J.M., et al., Microplasma discharge vacuum ultraviolet photoionization source for atmospheric pressure ionization mass spectrometry. Journal of The American Society for Mass Spectrometry, 2014. 25(9): p. 1557-1564. 